\title{
What is the Role of Race and Ethnicity in the Development Of Thionamide-Induced Neutropenia?
}

\author{
Mark D Ehrhart ${ }^{1}$, Iric R Guthrie ${ }^{1}$, Jose R Bucheli ${ }^{2}$, Mark R Burge ${ }^{1 *}$ \\ ${ }^{1}$ Department of Internal Medicine/Endocrinology, University of New Mexico, USA \\ ${ }^{2}$ Department of Economics, University of New Mexico, USA
}

Submission: November 14, 2017; Published: January 11, 2018

*Corresponding author: Mark R Burge, MD, Regents' and Distinguished Professor of Medicine, University of New Mexico Health Sciences Center, Department of Medicine/Endocrinology - 5ACC, MSC10-5550, 1 University of New Mexico, Albuquerque, NM, USA 87131-0001, Tel: (505)-272-4658 ; Fax: (505)-272-5155; USA, Email: mburge@salud.unm.edu

\section{Abstract}

Thionamides are anti-thyroid drugs (ATD) used to treat autonomous thyrotoxicosis. Although efficacious, these medications carry a risk of neutropenia or agranulocytosis. Some risk factors for ATD-induced neutropenia have been identified, including dose, age, and female sex, but the role of race and ethnicity has not been well studied. We hypothesize that there will be no effect of race or ethnicity on the change in Absolute Neutrophil Count (ANC) following initiation of ATD therapy.

Data from the Electronic Medical Record at UNM HSC were obtained using a standard database query. Inclusion criteria were the prescription of an ATD, an ANC recorded within 30 days of initiating ATD therapy (Pre-ATD), and an ANC recorded 75 - 365 days after starting an ANC (PostATD). Patients taking other agents known to cause neutropenia were excluded. Racial and ethnic groups were assigned as follows: Hispanic, Non-Hispanic White, Native American, Black/African American, and Asian/Pacific Islander. Post-ATD ANC was defined as the nadir ANC after ATD initiation. "Delta ANC" was defined as ((Post-ATD ANC) - (Pre-ATD ANC)). ANOVA analysis with Bonferroni-adjusted post-hoc testing and multiple regression were performed to examine differences in the mean changes in ANC across ethnic groups.

One hundred and twenty-three adult patients met inclusion and exclusion criteria and were included in the analysis. The Native American group showed a significantly greater Post-ATD ANC and Delta-ANC as compared to the other groups $(\mathrm{p}<0.001)$. In this cohort of New Mexicans with thyrotoxicosis, Native American race was protective against thionamide-induced neutropenia.

Abbreviations: ATD: Anti-Thyroid Drugs, Including Methimazole and Propylthiouracil; PTU: Propylthiouracil; ANC: Absolute Neutrophil Count; HLA: Human Leukocyte Antigen; GM-CSF: Granulocyte-Macrophage Colony Stimulating Factor; BMI: Body Mass Index; UNM HSC: University of New Mexico Health Sciences Center; WBC: White Blood Count; CLIA: Clinical Laboratory Improvement Amendments

\section{Introduction}

Treatment for hyperthyroidism often involves the use of anti-thyroid drugs (ATD) that inhibit thyroid peroxidase. These medications are also known as "thionamides." The only medications currently approved in this class in the United States are methimazole and propylthiouracil (PTU). Although uncommon, a dangerous side effect of these medications is neutropenia or agranulocytosis. Methimazole, a more potent drug, carries a greater associated drop in ANC than PTU [1]. Nevertheless, methimazole is more often used due to its once daily dosing and more rapid improvement in hyperthyroid symptoms [2]. In patients with severe neutropenia or agranulocytosis, a usually-benign presentation of fever and/or sore throat may signal a medical emergency. Agranulocytosis represents a small but significant morbidity and mortality risk. Estimates of the incidence of ATD-induced agranulocytosis are less than 1\%, and death is rare if the condition is recognized and treated [3].
The pathophysiology of ATD-induced neutropenia and agranulocytosis is not well understood. The most popular mechanistic hypotheses involve a combination of immunemediated and direct or indirect drug intoxication $[2,4,5]$. Immune-mediated destruction of circulating granulocytes is thought to be responsible for rapid-onset neutropenia and agranulocytosis, while direct or indirect drug intoxication is thought to be responsible for slower-onset neutropenia and agranulocytosis through suppression of granulopoiesis in the bone marrow.

Agranulocytosis typically presents in one of three ways: as asymptomatic, as asymptomatic evolving to symptomatic, or as symptomatic [3]. Asymptomatic agranulocytosis is detected on routine screening in patients without symptoms of fever or sore throat. Asymptomatic to symptomatic agranulocytosis is detected by screening patients who go on to develop symptoms despite 
treatment. Symptomatic agranulocytosis usually presents with a fever or pharyngitis that prompts White Blood Count (WBC) determination and investigation of granulocytopenia. More than $90 \%$ of patients who present with symptoms are febrile [6]. Agranulocytosis typically occurs between 2 and 12 weeks after initiating thionamidetherapy [7], but the condition may also occur after many months of apparently-safe therapy [8].

The effectiveness of complete blood count screening in patients beginning ATD therapy continues to be explored. There is some evidence to suggest that such screening is effective at identifying asymptomatic agranulocytosis, with two studies showing that most patients have a "slow onset" of agranulocytosis $[3,9]$. However, another study performed at the same hospital showed that more than $50 \%$ of patients who presented with symptomatic agranulocytosis had a normal granulocyte count within two weeks of presentation without treatment [10]. Thus, there is no consensus, and routine screening is not typically recommended [11].

Treatment for ATD-induced agranulocytosis involves removal of the offending medication, and beginning prophylactic antibiotics. In some cases, a combination of admission to the hospital, provision of an isolation room, blood transfusion, or administration of granulocyte/macrophage colony stimulating factor (GM-CSF) may also play a role in treatment $[3,7,12]$. Corticosteroids may also be beneficial, although there is disagreement on this $[7,13]$. The most frequently occurring infections are pharyngitis, tonsillitis, pneumonia, or urinary tract infection. Gram-negative rods are the most common offending organisms, including klebsiella pneumoniae and Pseudomonas aeruginosa. Thus, empiric antibiotic therapy for agranulocytosis should be broad in spectrum and include anti-pseudomonal coverage [6].

Known risk factors for agranulocytosis include thionamide dosage (more so for methimazole than with PTU), age, and female sex $[2,3,7,10,12]$. Other recent studies have used HLA typing to predict the risk for agranulocytosis. In China, HLA-B*38:02:01 was associated with a negative predictive value of 0.999 for agranulocytosis induced by methimazole or carbimazole (a methimazole derivative) [14]. Conversely, in Taiwan, this same HLA allele was found to carry an odds ratio of 21.48 for ATDinduced agranulocytosis, and the odds ratio rose to 48.41 rises if both this allele and HLA-DRB ${ }^{*} 08: 03$ were present [15]. These two studies were repeated in Europe, and another allele locus, HLA-B*27:05, was found to be the largest predictor of agranulocytosis among a white European population [16]. Despite this recent exploration into HLA typing, there are very limited data regarding how race and ethnicity impact the risk for ATD-induced agranulocytosis or neutropenia. Furthermore, many of the existing studies have been performed in Japan and China, and many of those studies were performed in the same Japanese institution [3,6,7,9,10,13-15,17]. Clearly, further studies of racial differences regarding this important adverse drug effect are needed [18].
Because treatment of thyrotoxicosis with ATD carries a finite risk of agranulocytosis, a better understanding of the role of race and ethnicity in its pathogenesis is necessary. The University of New Mexico Health Sciences Center (UNM HSC) is located in a geographically and ethnically diverse minority-majority state that allows convenient investigation of the potential relationship between race and ethnicity and the risk of ATD-associated neutropenia. We here describe a cross-sectional retrospective cohort study to explore how race affects change in absolute neutrophil count (ANC) in adult patients with thyrotoxicosis after initiation of an ATD. We hypothesize that there will be no effect of race or ethnicity on the change in ANC following initiation of ATD therapy.

\section{Methods}

\section{Participants}

Study participants were identified from the Electronic Medical Record at University of New Mexico (UNM) Health Sciences Center (HSC) using a standard database query for the years 2000-2016. Using an extant prescription for an ATD (i.e.methimazole or PTU), we identified 1,554 possible adult patients for inclusion in this study.

\section{Study protocol}

Inclusion criteria were adults aged 18 years or older, a prescription of an ATD during the study period, attainment of an ANC recorded within 30 days of initiating ATD therapy (Pre-ATD), and attainment of an ANC recorded between 75 and 365 days after initiating ATD therapy (Post-ATD). Exclusion criteria included prescription of other agents known to cause neutropenia or agranulocytosis, such as clozapine, allopurinol, and chemotherapy. Patients were assigned to self-reported racial and ethnic groups as follows: Hispanic, Non-Hispanic White, Native American, Black/African American, and Asian/ Pacific Islander. The date of ATD prescription was designated the "index date."Height, weight, and BMI were selected at the earliest available value within a period of 90 days before the index date to 365 days after, inclusive. ATD doses were classified high, medium, or low as follows: for Methimazole, low $<10 \mathrm{mg} /$ day (inclusive), medium $10-20 \mathrm{mg} /$ day (exclusive and inclusive, respectively), and high $>20 \mathrm{mg} /$ day (exclusive); for PTU, low $<100 \mathrm{mg} /$ day (inclusive), medium $100-300 \mathrm{mg} /$ day (exclusive and inclusive, respectively), and high $>300 \mathrm{mg} /$ day (exclusive). ATD doses were chosen as the prescription at the index date. Complete blood counts used to calculate ANC were obtained by standard phlebotomy and analyzed by the Clinical Laboratory Improvement Amendments (CLIA) certified Tricore Reference Laboratory (Albuquerque, NM) using standard clinical methodology.

\section{Outcome definitions}

The Pre-ATD ANC was selected as the value closest to the index date, with preference given to any ANC obtained before the index date. The Post-ATD ANC was defined as the nadir ANC 
observed between 75 and 365 days after the index date. "Delta ANC" was defined as the difference between Post-ATD ANC and Pre-ATD ANC ((Post-ATD ANC) - (Pre-ATD ANC)). Delta ANC was the primary study outcome variable. "Pre- to Post-ATD interval" was defined as the time between measurement of Pre-ATD ANC and Post-ATD ANC in days.

\section{Statistical analysis}

Baseline differences across racial groups were calculated. ANOVA analysis with Bonferroni-adjusted post-hoc testing was performed to examine differences in Delta ANC across ethnic groups, as well as Pre-ATD and Post-ATD ANC means. After Bonferroni adjustment, a probability of $<0.005$ was required for statistical significance. To further examine the independent role of race in risk for neutropenia with ATD administration, multiple regressions was performed after adjustment for age, sex, BMI, ATD dose category, and ATD to Post-ATD ANC interval. Stata software version 13.0 was used to perform all statistical analyses (Stata Corp 2013, College Station, TX). This study was approved by the UNM HSC Human Research Review Committee after expedited review.

\section{Results}

\section{Patient characteristics}

One hundred and twenty-three patients met inclusion and exclusion criteria and were included in the analysis. Specifically, 1,554 patients were identified as having an extant prescription for ATD. Of these, 1,103 patients were eliminated because they did not have an ANC value within 30 days of the index date (i.e. - they lacked a Pre-ATD ANC), and a further 303 were eliminated because they did not have an ANC between 75 and 365 days after the index date (i.e. - they lacked a Post-ATD ANC). Another 25 subjects were eliminated because they had prescriptions for other medications associated with neutropenia or agranulocytosis. The racial composition of the remaining 123 participants was 53\% Hispanic, 25\% Non-Hispanic White, 10\% Native American, 8\% Black, and 4\% Asian or Pacific Islander.

\section{Primary outcome}

Provides descriptive characteristics, dose category, and ANC outcomes of the 123 participants by racial group. The comparison of Delta-ANC across ethnic groups using ANOVA indicates that there is a significant effect of ethnicity at the $p<0.001$ level for the five groups $(F(4,115)=5.52, p=0.0004)$. Further comparison using Bonferroni's post-hoc adjustment suggests that the mean Delta-ANC for Native Americans $\left(3.6 \pm 5.1 \times 10^{\wedge} 3\right.$ permm $\left.^{\wedge} 3\right)$ is significantly different as compared to all other ethnic groups (Hispanic $=-1.4 \pm 3.3$, White $=-0.6 \pm 3.3$, Black $/$ African American $=-0.9 \pm 4.1$, Asian/Pacific Islander $=-3.8 \pm 4.8$ ). There was no statistically significant difference between the groups with regards to age, sex, BMI, ATD dose category, Pre-ATD ANC, or Pre- to Post-ATD ANC interval.

Multiple regression, which controlled for age, sex, BMI, ATD dose category, and ATD to Post-ATD ANC interval, confirmed this finding $\left(\mathrm{r}^{\wedge} 2=0.23, \mathrm{p}=0.0077\right)$. The Native American group was the only racial and ethnic group to record, on average, a positive Delta-ANC, signifying an overall increase in ANC after initiating an ATD. Figure 1 further illustrates this change, displaying DeltaANC as a function of time.

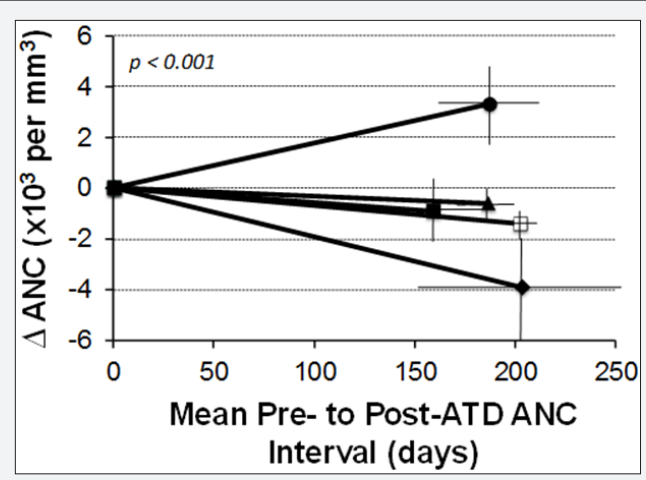

Figure 1: Change in Absolute Neutrophil Count $(\triangle A N C)$ plotted against the time interval between the attainment of Pre-ATD ANC and Post-ATD ANC according to racial or ethnic group. Native American (solid circle), Non-Hispanic White (solid triangle), Black (solid square), Hispanic (vacant square), and Asian or Pacific Islander (solid diamond).Error bars represent standard error of the mean.

\section{Discussion}

In this retrospective cohort study, we examined how race and ethnicity affected the risk of neutropenia and agranulocytosis among unselected patients who were receiving anti-thyroid drug therapy and who conveniently had Complete Blood Counts obtained at appropriate times to assess the effect of these medications on neutropoiesis during the first year of therapy. We found that Native American race was independently protective against development of neutropenia, even after adjusting for the potential confounders of age, sex, ATD dose, and BMI.

Our study has several strengths. First, this study addresses an important clinical question of how race and ethnicity affect the risk for neutropenia and agranulocytosis after initiating ATD therapy. While there have been other recent publications exploring the relationship of HLA typing to the risk of neutropenia, a simple investigation of the role of race in ATDinduced neutropenia has not, to our knowledge, been previously documented [14-16]. Thus, this study addresses an important gap in the medical literature. Second, UNM HSC is uniquely positioned within the Southwestern United States as a major referral center for Endocrinology care, and the availability of a large, multiethnic population likely increases the generalizability of our study. Specifically, New Mexico's ethnic and racial makeup in 2014 was approximately 45\% Hispanic, 40\% Non-Hispanic White, 9\% Native American, 5\% Asian or Pacific Islander, and $1 \%$ Black [19].

There are also limitations to this study. First, it should be noted that the racial or ethnic designation of "Native American" encompasses a large and diverse population. Our study likely contains a high percentage of Native Americans from the New 
Mexican Pueblos, and thus our results may not be generalizable to all Native American communities across the United States. Second, the sample size from this study is relatively small. This is due to a relative paucity of Pre-ATD ANC data, as 71\% (1103 of 1554) of our possible ATD patient pool was excluded because of an absence of necessary ANC data. This likely reflects the lack of current guidelines mandating the monitoring of WBC in patients who start ATD therapy. Moreover, a further 19\% of our potential study subjects (303 of 1554) were excluded because they lacked a Post-ATD ANC value. This may again reflect the current standard of practice, which does not include regular monitoring of WBC among patients receiving ATD therapy. Additionally, patients who did receive ATD determination in this study presumably did so for some clinical indication, and those reasons were not captured by our study design. There is additional evidence to suggest that hyperthyroidism is more prevalent in Non-Hispanic black racial groups, and that the prevalence of hyperthyroidism is approximately equal among Hispanic and Non-Hispanic whites [20]. It is also worth noting that the Black/African American population of New Mexico is relatively small, so firm conclusions about the effect of ATDs on ANC in African American patients probably cannot be drawn from this study [19]. Additionally, ANC values were chosen that most closely corresponded to a true "Pre-ATD" and "Post-ATD" timeline, yet ANC values for "Pre-ATD" were sometimes selected after (but within 30 days of) the date of initiation of the ATD. Since the neutropenic effect of an ATD can happen any time after initiation, this may have confounded our results $[7,8]$. Finally, blood counts and ANC can vary from day to day. Our study design selected a single ANC measurement to be representative of the patient's true ANC status, which may or may not have been an accurate characterization. Despite these limitations, we took a conservative approach to statistical analysis by employing the Bonferroni-adjustment to our interpretation results.

Future research should expand upon the current study to confirm these findings with larger and more diverse national populations, and a prospective study investigating the relationship between race and ethnicity on ATD-associated neutropenia risk is sorely needed. We conclude that in this cohort of New Mexican hyperthyroid patients, Native American individuals had a decreased risk of developing neutropenia than other racial groups following initiation of an anti-thyroid medication in the thionamide class. This finding provides important further characterization of the known risk factors for this potentially life-threatening side effect of anti-thyroid therapy.

\section{Acknowledgement}

The authors wish to thank Laurie McPherson of the Clinical \& Translational Science Center for her work in providing the data from the electronic medical records.

\section{Disclosure Statement}

The authors have no conflicts of interest to disclose

\section{Funding}

This study was supported by the University of New Mexico Clinical and Translational Research Center (NCATS \#8UL1TR000041).

\section{References}

1. Kim HK, Yoon JH, Jeon MJ, Kim TY, Shong YK, et al. (2015) Characteristics of Korean Patients with Antithyroid Drug-Induced Agranulocytosis: A Multicenter Study in Korea. Endocrinol Metab (Seoul) 30(4): 475-480.

2. Cooper DS (2005) Antithyroid Drugs. N Engl J Med 352(9): 905-917.

3. Tajiri J, Noguchi S, Murakami T, Murakami N (1990) Antityhroid druginduced agranulocytosis. The usefulness of routine white blood cell count monitoring. Arch Intern Med 150(3): 621-624.

4. Bhatt V, Saleem A (2004) Review: Drug-induced neutropenia pathophysiology, clinical features, and management. Ann Clin Lab Sci 34(2): 131-137.

5. Fibbe WE, Claas FH, Van der Star-Dijkstra W, Schaafsma MR, Meyboom RH, et al. (1986) Agranulocytosis induced by propylthiouracil: evidence of a drug dependent antibody reacting with granulocytes, monocytes and haematopoietic progenitor cells. Br J Haematol 64(2): 363-373.

6. Sheng WH, Hung CC, Chen YC, Fang CT, Hsieh SM, et al. (1999) Antithyroid-drug-induced agranulocytosis complicated by lifethreatening infections. QJM 92(8): 455-461.

7. Dai WX, Zhang JD, Zhan SW, Xu BZ, Jin H, et al. (2002) Retrospective analysis of 18 cases of antithyroid drug (ATD)-induced agranulocytosis. Endocr J 49(1): 29-33.

8. Mutharasan P, Oatis W, Kwaan H, Molitch M (2012) Delayed antithyroid drug-induced agranulocytosis. Endocr Pract 18(4): e69-e72.

9. Nakamura H, Ide A, Nishihara E, Ito M, Miiyauchi A (2016) Periodic Granulocyte Count Measuring Is Useful for Detecting Asymptomatic Agranulocytosis in Antithyroid Drug-Treated Patients with Graves' Disease. Eur Thyroid J 5(4): 253-260.

10. Nakamura H, Miyauchi A, Miyawaki N, Imagawa J (2013) Analysis of 754 cases of antithyroid drug-induced agranulocytosis over 30 years in Japan. J Clin Endocrinol Metab 98(12): 4776-4783.

11. Bahn RS, Burch HB, Cooper DS, Garber JR, Greenlee MC, et al. (2011) Hyperthyroidism and other causes of thyrotoxicosis: Management guidelines of the American Thyroid Association and the American Association of Clinical Endocrinologists. Thyroid 21(6): 593-646.

12. Meyer-GM, Benker G, Lederbogen S, Olbricht T, Reinwein D (1994) Antithyroid drug-induced agranulocytosis: clinical experience with ten patients treated at one in stitution and review of the literature. J Endocrinol Invest 17(1): 29-36.

13. Hamada N, Itoh K, Mototani N, Nishikawa Y, Mimura T, et al. (1981) Effect of corticosteroid in 10 cases of methimazole-induced agranulocytosis. Endocrinol Jpn 28(6): 823-827.

14. Cheung CL, Sing CW, Tang CS, Cheng VK, Pirmohamed M, et al. (2016) HLA-B*38:02:01 predicts carbimazole/methimazole-induced agranulocytosis. Clin Pharmacol Ther 99(5): 555-561.

15. Chen PL, Shih SR, Wang PW, Lin YC, Chu CC, et al. (2015) Genetic determinants of antithyroid drug-induced agranulocytosis by human leukocyte antigen genotyping and genome-wide association study. Nat Commun 6: 7633.

16. Hallberg P, Eriksson N, Ibañez L, Bondon-GE, Kreutz R, et al. (2016) Genetic variants associated with antithyroid drug-induced agranulocytosis: a genome-wide association study in a European population. Lancet Diabetes Endocrinol 4(6): 507-516. 
17. Tajiri J, Noguchi S, Okamura S, Morita M, Tamaru M, et al. (1993) Granulocyte colony-stimulating factor treatment of antithyroid druginduced granulocytopenia. Arch Intern Med 153(4): 509-514.

18. Hamerschlak N, Maluf E, Biasi CA, Avezum JA, Eluf-NJ, et al. (2008) Incidence and risk factors for agranulocytosis in Latin American countries--the Latin Study: a multicenter study. Eur J Clin Pharmacol 64(9): 921-929.
19. Health Indicator Report of New Mexico Population Demographics Race and Ethnicity (2014). New Mexico's Indicator-Based Information System (NM-IBIS), New Mexico Department of Health, USA.

20. McLeod DS, Cooper DS, Ladenson PW, Whiteman DC, Jordan SJ (2015) Race/Ethnicity and the prevalence of thyrotoxicosis in young Americans. Thyroid 25(6): 621-628.

\section{Your next submission with Juniper Publishers} will reach you the below assets

- Quality Editorial service

- Swift Peer Review

- Reprints availability

- E-prints Service

- Manuscript Podcast for convenient understanding

- Global attainment for your research

- Manuscript accessibility in different formats ( Pdf, E-pub, Full Text, Audio)

- Unceasing customer service

Track the below URL for one-step submission https://juniperpublishers.com/online-submission.php 